\title{
LBS AS VECTORS OF INFLUENCE
}

\author{
Maximilian Gartner ${ }^{\text {a,* }}$

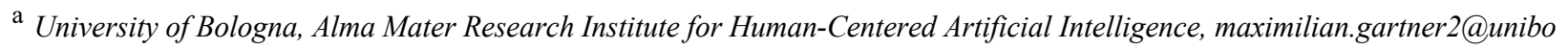 \\ * Corresponding author
}

\begin{abstract}
Due to their technical capabilities and use-cases, LBS can impose autonomy constraints on individuals, both through direct interaction and via indirect means. This essay outlines four main interaction characteristics that constitute LBS as a vector of influence and contextualizes them by mapping them onto a pragmatic framework of autonomy.
\end{abstract}

Keywords: Autonomy, Privacy, LBS, Persuasive Technology, Nudging

\section{Introduction}

Humans are increasingly interacting with algorithmic agents belonging to the class of context-aware services; this includes interaction with processes that can be identified as pertaining to location-based-services (LBS). These interactions are not necessarily autonomy-neutral, i.e. as a result of such interaction, the affected individual may experience constraints to their capability of selfdetermination with respect to some aspects of their life for a certain period of time. Put differently, LBS can become a vector of influence and a specimen of so-called "persuasive technology". Research into the impact of technology on human autonomy has intensified recently, both generally (Calvo et al. 2020), and with respect to specific technology uses that are LBS-adjacent such as in the personal health domain (Owens and Cribb 2019). Still, the technical, ethical and legal challenges of many fields of technology remain underexplored in current discourse (Mittelstadt et al. 2016; Tsamados et al. 2021) and this holds true in particular with respect to LBS. A brief investigation of this phenomenon is the purpose of this essay.

\section{Fundamentals, Scoping and Terminology}

Understanding the impact of LBS on human autonomy requires scoping both the terms autonomy and LBS, as well as cursory understanding of the relevant domain, in which interactions and influence between individuals and LBS take place, i.e. a sphere characterized by almost ubiquitous computing / omnipresent data collection and processing (Weiser 1999). Hence, this section outlines the general direction of inquiry undertaken in this essay.

\subsection{Autonomy}

This inquiry concerns human autonomy, i.e. the capacity and exercise of self-governance of individuals. Autonomy in this context is primarily a term subject to stipulative definition that more or less tracks the intuition of the theorists outlining it. Different models are under consideration in current scholarly debate. One of the most impactful set of theories belonging to the class of hierarchical autonomy theories considers an individual's preferences, desires, decisions etc. (i.e. mental states), their hierarchy, their congruence with each other and their effectiveness in influencing the individual's action. Simplified, autonomy is thought to be achieved if an individual's actions are effectuated by mental states that are in conformity with other, higher mental states (Frankfurt 1971; Sneddon 2013; Dworkin 2015). A second school of thought belonging to the class of relational autonomy theories considers instead the connections and relationships of an individual to other individuals, entities and their environment to be either relevant or constitutive to an individual's autonomy (Brison 2000; Stoljar 2000; Benson 2005).

Of interest is how autonomy constraints are connected to the technical characteristics, utilization, interface, and informational architecture with respect to human-relevant output of LBS. Aforementioned autonomy theories are internally consistent, but generally not compatible with each other. Indeed, intuitive concerns by scholars (Mittelstadt et al. 2016; André et al. 2018), theorists (Zuboff 2019) or other stakeholders (Special Rapporteur on the promotion and protection of the right to freedom of opinion and expression 2018; Council of Europe 2019) that pertain to autonomy in tension with algorithmic agents do not always fit neatly into existing doctrine. In other words, much of the current discourse about risks to individual autonomy may not be tracked under a specific autonomy theory, but fueled by intuitive understanding (and colloquial use) of the term and concept of autonomy. It is useful then to understand the concept in a more encompassing way than existing (philosophical) theories suggests. For this purpose I here use a tripartite model, the 
pragmatic account of autonomy, that is partly derived from these existing theories but aims to track widespread intuitive concerns more holistically. I develop this model elsewhere in more detail (Gartner 2021), but the following paragraphs will give a brief overview of this model.

Within the pragmatic account of autonomy, we can consider three dimensions, i.e. aspects, of individual autonomy that are most salient to understanding constraints imposed by LBS: (1) intrinsic autonomy, (2) informational autonomy and (3) relational autonomy. An individual has a status of diminished autonomy if they are constrained on one or more of these dimensions.

Intrinsic autonomy describes the exercise of autonomy, or constraints hereto that is connected to the internal endorsement of an individual's mental states (e.g. preferences, volitions, etc.) by other (potentially higherorder) mental states and their realization. Here, autonomy constraints are imposed if the congruence of mental states is subjected to externally introduced imbalance, and/or if new mental states are inserted by external factors or entities and are not sufficiently integrated into the overall system of the individual's mental states. For example, highly effective advertising feeding on location data may introduce, i.e. insert, a want to consume a certain good in an individual. This want, a mental state by itself, may then be in conflict with other (competing) wants to consume other (competing goods) or the want to not consume any goods, with this conflict already diminishing the individual's autonomy. In addition, the individual may have a higher-order mental state that references this class of mental states; the individual may not want to want to consume the advertised good. Is the advertising successful in overriding the other competing wants and does the individual take action in conflict with their higher order want to not consume, we may consider the intrinsic autonomy of the individual diminished with respect to the taken action.

Informational autonomy is concerned with information and its disclosure. Constraints are imposed in particularly by missing, misleading or false information, or alternatively by an overload of information with respect to actions/decisions an individual may take or with respect to a constraint that is imposed on the individual within a different dimension of the pragmatic model. If an individual is not aware of an algorithmic agent tracking their location, the individual may not be able to act upon that knowledge in any way; the same being true if the individual is being misled about said tracking.

Relational autonomy is constituted and constrained by an individual's relation to other entities, their environment and factual constraints. Most importantly, constraints on this dimensions are dividable into factual, normative or societal constraints, while also belonging to the class of either physical or mental constraints. An individual that interacts with a LBS device through an interface that is designed with an individual's likely cognitive biases in mind to steer them towards certain actions may experiences mental (factual) constraints on their autonomy, in addition to a likely constraint on their informational autonomy. I will return to some of these autonomy dimensions again later when discussing LBShuman interactions.

\subsection{Location Based Services (LBS)}

The term Location Based Services (LBS) broadly describes the combination or integration of location information with other types of services, although there seems to be no full consensus on an exact definition (Schiller and Voisard 2004, p. 1; Raper et al. 2007a). While the concept of LBS used to primarily denote applications for the explicit purpose of navigation or their directly adjacent uses such as mobile guides or navigation systems (Raper et al. 2007b), the field of relevant LBS applications has become more complex in the recent years. LBS are now interwoven with applications in many different domains both serious, such as disaster and emergency context, or recreational, such as gaming or fitness (Huang et al. 2018). This mirrors are larger trend of technological processes becoming both more capable and widespread. This essay uses the term LBS device mostly with reference to devices that have capabilities to determine their geospatial location themselves, e.g. via use of GNSS. However, it may be advantageous to understand the term LBS as to denote algorithmic agents that deal with location data in a wider sense, or further down into the information supply chain, e.g. a service that processes location data but does not collect or affirm it itself. Such wide terminology necessarily softens the scope of what ought to be called a LBS device as a consequence. While data processing is increasingly done not only on-device but server-side, this allows the use of LBS on an increasing number of devices, so long as they have interconnectivity capabilities. In this sense it may be useful to differentiate between core LBS devices that can determine their own geospatial location and other LBS devices that cannot, but are still able to provide LBS.

\subsection{Interconnectivity and Device Capability}

The increasing prevalence of LBS is aided by the adoption of devices capable of creating and processing relevant location data and of an improvement of existing infrastructure. Mobile phones, a class of devices that tend to have LBS-capabilities, have become a major access point for many people as their use continues to rise (Pew Research Center 2019). At the same time, there is an 
increasing amount of devices that add LBS-capabilities to their existing set of primary features such as fitness heartrate monitors or "smart-watches" that may receive GNSS information or have navigational capabilities, with these devices being considered as being part of the "Internet of Things".

Interconnectivity allows interaction on the basis of an individual's location data, thereby multiplying the respective use cases of said data beyond mere confirmation of location. To this end, the increasing adoption of LBS is also supported by a consistent improvement of Internet infrastructure as displayed by continuously increasing bandwidth (International Telecommunication Union 2020), as characterized in the observation called "Edholm's Law" (Cherry 2004). This holds true especially for wireless interconnectivity which is improving at a faster rate than wired connections (Rappaport 1991; Ericsson 2021), adoption of which further favors the mobile nature of LBS-devices.

\subsection{Data and Information}

An LBS device may passively receive information about its location, process this information offline, i.e. on the device, and then proceed by exercising its programmed function. For example, a satellite navigation device used for car-based navigation may conduct its service, i.e. geospatial positioning and consequent route-finding, based on map data that is stored on the device. The device may collect and store, i.e. log, part of this information even after the individual service is provided, e.g. to display a list of "last-used destinations" for the convenience of the user, but that information is likely to remain on the device. This makes the data collected of little use for the provider of an LBS. However, as pointed out above, many LBS devices do now possess interconnective capabilities and location data is used in many more ways than route finding. As LBS are now often embedded into other services that also rely on interconnectivity, e.g. social media, communication, etc., data collected on the device can now be shared (back) with the LBS provider, allowing the analysis of said data irrespective of the device's capabilities or the user's active participation. The increase in capability of LBS devices, use and use cases of LBS, and capability of interconnective infrastructure together with improvements in data analysis suggests that individuals leave behind "data trails" that are both more comprehensive in a quantitative sense as well as more informative generally. This likely creates an economic incentive to maximize access to such information (Zuboff 2015), which in turn may conflict with the individual's privacy (Cvrcek et al. 2006; Wicker 2012; Cheung 2014). Of course, privacy is generally seen as inextricably linked or even constitutive of autonomy (Benn 1984; Kupfer 1987; Tsamados et al. 2021). At the same time, increased data acquisition may also be motivated by improving the LBS via collection and analysis of non-location based information as context (Schmidt et al. 1999; Yurur et al. 2016; Grifoni et al. 2018). This positions LBS as a dualistic phenomenon with respect to data: LBS and their immediate location data collection and analysis processes may be incorporated into other information processing routines for context; at the same time LBS also benefits from additional non-location data for context all the same.

\subsection{Vectors of Influence}

Combining the phenomena described above, I suggest here the use of the term vector of influence to better conceptualize autonomy constraints imposed by LBS. I define this concept as follows: a vector of influence in $a$ narrow sense is the compound phenomena that characterize a given interaction between an algorithmic agent and another entity that is capable of imparting change onto an individual's autonomy; a vector of influence in a wide sense describes the algorithmic agent, its procedural and physical structure and its capabilities (including the vectors of influence in a narrow sense at its disposal) to impart change onto an individual's autonomy more generally. An LBS device outfitted with an algorithmic agent that provides LBS may be a vector of influence in a wide sense. The type of information output, the design and characteristics of the output and interaction interface and the context of use of the LBS device describe the vector of influence in a narrow sense.

Having established that LBS and the respective LBS devices are increasing in capability, connectivity and widespread use, it becomes obvious that the LBS are (or may utilize) more and more effective vectors of influence onto an individual's autonomy.

\section{Interaction Characteristics}

Understanding potential autonomy constraints imposed by LBS as aforementioned vectors of influence allows a closer analysis of the interaction between LBS and individuals. LBS-human interaction can be characterized on different dimensions. Most fundamentally, LBS can affect an individual's autonomy twofold. The LBS may interact with the individual, i.e. the user, directly, by using some sort of interface of transmitting information between the LBS agent and the user. This will encompass most if not all LBS that are consciously enabled by the user for the purposes of benefitting directly from the LBS. For example, a navigation system outputting route-finding information interacts with an individual directly via (audio-) visual outputs. However, an LBS may also be non-interactive from the side of the affected individual. Contextual location data may be collected by an 
application of a LBS-capable device that does not benefit the individual directly, or at all. Often, there may be informational friction, with the individual not in full control or knowledge about the data collected at any given time. LBS that belong to the class of agents most removed of user-interaction are those that assign data to the individual for the purposes of profiling.

These differences already make visible the variance of autonomy constraints that can be imposed by LBS. Interactive LBS can use similar vectors of influence as physical entities including other individuals when imposing autonomy constraints such as nudging, or deception. Non-interactive LBS are instead more likely to constrain an individual on an informational perspective, given that their data processing and assignation happens not (only) for the purposes of disclosing the resulting information back to the affected individual. However, this may not be the full picture. If assignation of data by the LBS or a subsequent algorithmic agent leads to other entities making decisions affecting the individual, e.g. effectively preventing the individual to take certain decisions on the basis of the data collected by the LBS, the individual may be relationally, more precisely factually constrained. A LBS that observes a geofencing violation by the individual and cause a different entity to disable the individual's access to certain services or actions (e.g. entering a different controlled location) constrains the individual's autonomy without interacting with them directly.

More systematically I suggest that the following interaction characteristics are of particular interest and relevance: (1) the medium of information transfer and its characteristics, (2) the perceived accuracy, precision, trustworthiness, and assertiveness of information, (3) transparency to the user with respect to the information collection process of the LBS and alternative action potentials available to the user (where applicable), and (4) the assignation of actionable data to a human user. The final part of this essay will shortly elaborate on these aspects as they pertain to potential autonomy constraints.

\subsection{Medium of Information Transfer}

Interaction between an LBS algorithmic agent and an individual are necessarily intermediated, usually through some type of interface. The imparting of information may be conducted e.g. via audio-visual or haptic cues. In particular, visual interfaces may engage with a multitude of known biases, with respect to both perception and cognition that the user may display (Wall et al. 2019), the most obvious of which is the implicit or explicit ranking of the displayed information; a choice likely to influence the user (Taib 1990; Friedman and Nissenbaum 1996). Interfaces may also be designed deliberately hostile to increase mental load of the user, which in turn may make them more susceptible to biases (Allred et al. 2016). In general, information transfer is conducted in a way that follows the design of the LBS' front-end. To the extent it is interactive, the LBS interface may be understood as an example of choice architecture, i.e. a decisional infrastructure "organizing the context in which people make decisions" (Thaler et al. 2013). Of course, choice architecture is generally capable of altering individual's behavior to a certain extent, i.e. the aforementioned nudge (Kosters and Van der Heijden 2015). This is potentially even more effective if the choice architecture is personalized via the data collected on the user (Tene and Polonetsky 2013). Using the language of this essay, the medium of information transfer constitutes a vector of influence that may constrain an individual's autonomy in particular on their informational dimension, e.g. via informational overload in case of hostile design, and on their relational dimension, in particular by imposing factual and mental constraints, e.g. by the exploitation of cognitive or perceptual biases. At the same time, very effective (and potentially value-laden) interface design may qualify as an intrinsic autonomy constraint, as it may insert or affect the individual's mental states, e.g. via creating an assumption of value through the display of ranked information or context of social value, whereas these impart influence on the individual's preferences.

\section{2 (Perceived) Veracity, Accuracy, Trustworthiness and Assertiveness}

Interaction between LBS agents and other entities (including their users) consists of information transfer; it follows that the quality of that information is of paramount relevance to the receiver (Lee 2018). Inaccurate or misleading output of LBS is likely to constrain an individual on their informational autonomy, as they must exercise their autonomy on the basis of faulty information. This issue is compounded by the fact that individuals may display deference to algorithmic decision-making (Bogert et al. 2021). However, this may be mitigated by creating accurate expectations of the receiver with respect to the information transferred. For example, the LBS may disclose information about the quality of the output, e.g. a human-readable confidence score which can prevent nonjustified reliance on the LBS in question.

Intrinsic autonomy constraints may be imposed by an overly effective or assertive LBS. Algorithmic agents may insert preferences or information into the individual's decisional structure (de Vries 2010; Burr et al. 2018).

\subsection{Transparency and Completeness of Information}

The interaction of algorithmic agents and individuals can be characterized by an information asymmetry. The 
affected individual may lack information about the data collection and analyzing processes they are subjected to, and the consequences that may entail, which constitutes an informational autonomy constraint. Lack of transparency may also undermine the trust of individuals into the LBS (Sloan and Warner 2018), which can impede individuals consciously using LBS to exercise their autonomy. Similar constraints may also be incurred in the context of LBS as recommender-esque systems, e.g. as route-finding applications. In connection with LBS deference as discussed above, lack of information about other viable recommendations, e.g. an alternative but equally viable route that is not displayed on a navigational device, constrains the individual in their ability to autonomously choose an alternative action, e.g. the alternative route. Indeed, limiting exposure to potential venues of autonomy exercise imposes relational-factual constraints in addition to any informational constraints, and is a well-recognized source of diminished autonomy (Koene et al. 2015; Taddeo and Floridi 2018).

\subsection{Assignation of actionable data}

As pointed out in Section 2.4, LBS may collect significant amount of data; this data may be used to gain further (predictive) insights into the respective individual and their behavior beyond mere location data (Bilal et al. 2019), a practice known as profiling. These profiles may then be used by other entities to make decisions affecting the individual; such as excluding them from purchasing certain products or enjoying certain services, e.g. in the field of insurance (Cevolini and Esposito 2020); in a more intensive form the LBS may become part of a social scoring system (Hoffrage and Marewski 2020). These environments tend to constrain individuals on their relational-factual dimension, by imposing real-life limits onto the action potential of a person.

\subsection{Special Case: Inhibition}

Lastly, there be may an additional intrinsic and/or relational-mental autonomy constraint imposed by the fact that individuals have knowledge of LBS collecting data on them. Individuals may be discouraged from using certain services based on concerns about their information exposure, e.g. for reasons of privacy, and this holds true for LBS (Xu and Gupta 2009). Inhibition with respect to certain actions, e.g. using a certain LBS device in a certain way in a certain context, to avoid embarrassing or incriminating data to be collected, may steer individuals' decision making. This may lead to the individual avoid using the LBS device in certain locations. However if this is inconvenient, e.g. mobile phones that are used for a variety of reasons incur convenience costs when turned off or left behind, or impossible, e.g. LBS devices that are permanently installed into vehicles that the individual must use, the individual may choose to alter their behavior in order to avoid the undesired data collection by the LBS device, creating intrinsic and/or relational-societal/mental autonomy constraints.

\subsection{Positive Vectors of Influence}

Imparting influence or other effects on an individual by an algorithmic agent such as an LBS is not necessarily harmful to one's autonomy. Indeed, like other services, LBS can assist an individual in exercising their autonomy by enabling the individual to pierce through existing constraints. We can consider this in all three dimensions of autonomy.

Enabling the exercise of "true interests", that is mental states that have been reflected and integrated into the identity of an individual sufficiently, and excision of lower-order preferences that are in contradiction to said true interests can be seen as strengthening an individual's intrinsic autonomy. An individual that deals with unwanted compulsion to consume unhealthy food may be benefitted by an LBS that recognizes their pathing towards fast-food restaurants and interacts, e.g. by notification on their phone that discourages them via some sort of effective messaging from the undesirable behavior. In this way, an incongruent mental state, that may be created internally in the individual but causes conflict and hence diminishes intrinsic autonomy, is counteracted.

Informational autonomy may be supported by overcoming the constraints caused by lack of informational quality or by helping an individual parse large amounts of information. An LBS interface that is designed to take off mental load from the interacting individual by ranking the displayed information in a way that the individual is not influenced to take actions that are not in their interest has avoided or minimized the informational/mental autonomy constraints of information overload and search costs. Indeed, recommender systems of all types suffer from a sort of principal-agent paradox, in which the interests of the provider and the information recipient are not necessarily aligned (Hosanagar et al. 2008). The closer the results of the recommender systems are aligned with the true interests of the users, the more likely they are to help curb informational autonomy constraints; the more these systems reflect other interests to the detriment of the true interest of the users, the more likely they are to impose informational autonomy constraints. In addition, such systems may have implications for third parties as well; LBS may have tacit stakeholders that benefit or depend on the recommender system for their exercise of autonomy (Milano et al. 2020).

Closely related to informational interventions, transparency-related design choices of the LBS can also 
assist an individual's relational autonomy. As discussed above, lack of information about potential actions with which autonomy can be exercised imposes not only an informational but also a relational-factual constraint. The individual is not just constrained because they do not know but also because based on the lack of knowledge they cannot act in a certain way. To this end, the enabling of providing information about alternative routes, e.g. based on speed, traffic, road types and fuel consumption (Glasgow 2021), help unlock decisions that the individual was not able to take before learning the relevant information.

Inhibition of individuals to exercise their autonomy freely because of concerns related to the algorithmic observation that LBS entail, a relational-mental constraint of sorts, may be counteracted by establishing trust into the algorithmic agent that provides LBS, which leads to increased satisfaction (Shin and Park 2019) and exercise their autonomy with respect to data collection and processing that may impede or support their autonomy in the future (Sloan and Warner 2018). Ultimately however, the proper balance between technology supporting and constraining exercise of autonomy, i.e. how many decisions and actions are delegated to LBS may hard to ascertain generally (Ananny and Crawford 2018; Floridi et al. 2018; Tsamados et al. 2021).

\section{Conclusion}

This essay has outlined the concept of LBS as a vector of influence. Just like other context-aware services and technology at large, LBS is not necessarily autonomy neutral. Individuals may be constrained in their autonomy when interacting with, or being subject to the processing of an LBS or respective LBS device. Accepting this, it becomes a necessary conclusion that due to the change to LBS use and capability, LBS' potential to affect an individual's autonomy is increasing. This is relevant, as autonomy itself is generally not considered ethically neutral but omnirelevant, i.e. worth protecting (Sneddon 2013). Hence constraints to an individual's autonomy are considered ethically problematic (insofar no other interests prevail). It follows that LBS, like other algorithmic agents (Martin 2019), insofar they are imposing autonomy constraints, are not (considered to be) ethically neutral either. It seems prudent then to consider the implications of LBS for individual autonomy at all stages of design and deployment. The outlined areas of particular interest, such as the assertiveness of information or transparency of data collection and analysis processes, represent but a starting point, in which future (technical and empirical) research may be contextualized through the lens of autonomy considerations.
In conclusion, this essay is intended to help sensitize involved stakeholders and researchers alike to the significance of autonomy considerations in the context of LBS. The increase of LBS' capability and use likely add value to the individuals affected by their respective vectors of influence. At the same time, the benefits of such algorithmic agents make questioning their implications for human autonomy ever more salient. To this purpose, the present essay is but a first primer.

\section{Acknowledgements}

This project has received funding from the European Union's Horizon 2020 research and innovation programme under the Marie Skłodowska-Curie ITN EJD grant agreement No 814177.

\section{References}

Allred SR, Crawford LE, Duffy S, Smith J (2016) Working memory and spatial judgments: Cognitive load increases the central tendency bias. Psychon Bull Rev 23:1825-1831. https://doi.org/10.3758/s13423-016-1039-0

Ananny M, Crawford K (2018) Seeing without knowing: Limitations of the transparency ideal and its application to algorithmic accountability. New Media Soc 20:973-989. https://doi.org/10.1177/1461444816676645

André Q, Carmon Z, Wertenbroch K, et al (2018) Consumer Choice and Autonomy in the Age of Artificial Intelligence and Big Data. Cust Needs Solut 5:28-37. https://doi.org/10.1007/s40547-0170085-8

Benn S (1984) Privacy, freedom, and respect for persons. In: Shoeman F (ed) Philosophical Dimensions of Privacy: An Anthology. Cambridge University Press, Cambridge

Benson P (2005) Feminist intuitions and the normative substance of autonomy. In: Taylor J (ed) Personal Autonomy: New Essays on Personal Autonomy and Its Role in Contemporary Moral Philosophy. Cambridge University Press, Cambridge, pp 124142

Bilal M, Gani A, Lali MIU, et al (2019) Social Profiling: A Review, Taxonomy, and Challenges. Cyberpsychology, Behav Soc Netw 22:433-450. https://doi.org/10.1089/cyber.2018.0670

Bogert E, Schecter A, Watson RT (2021) Humans rely more on algorithms than social influence as a task becomes more difficult. Sci Rep 11:8028. https://doi.org/10.1038/s41598-021-87480-9

Brison S (2000) Relational autonomy and freedom of expression. In: Mackenzie C, Stoljar N (eds) Relational Autonomy: Feminist Perspectives on Autonomy, Agency and the Social Self. Oxford University Press, pp 280-300 
Burr C, Cristianini N, Ladyman J (2018) An Analysis of the Interaction Between Intelligent Software Agents and Human Users. Minds Mach 28:735774. https://doi.org/10.1007/s1 1023-018-9479-0

Calvo RA, Peters D, Vold K, Ryan RM (2020) Supporting Human Autonomy in AI Systems: A Framework for Ethical Enquiry. pp 31-54

Cevolini A, Esposito E (2020) From pool to profile: Social consequences of algorithmic prediction in insurance. Big Data Soc 7:205395172093922. https://doi.org/10.1177/2053951720939228

Cherry S (2004) Edholm's law of bandwidth. IEEE Spectr 41:58-60. https://doi.org/10.1109/MSPEC.2004.1309810

Cheung ASY (2014) Location privacy: The challenges of mobile service devices. Comput Law Secur Rev 30:41-54. https://doi.org/10.1016/j.clsr.2013.11.005

Council of Europe (2019) Declaration by the Committee of Ministers on the manipulative capabilities of algorithmic processes

Cvrcek D, Kumpost M, Matyas V, Danezis G (2006) A study on the value of location privacy. In: Proceedings of the 5th ACM workshop on Privacy in electronic society - WPES '06. ACM Press, New York, New York, USA, p 109

de Vries K (2010) Identity, profiling algorithms and a world of ambient intelligence. Ethics Inf Technol 12:71-85. https://doi.org/10.1007/s10676-0099215-9

Dworkin G (2015) The nature of autonomy. Nord J Stud Educ Policy 2015:28479. https://doi.org/10.3402/nstep.v1.28479

Ericsson (2021) Mobility Report

Floridi L, Cowls J, Beltrametti M, et al (2018) AI4People-An Ethical Framework for a Good AI Society: Opportunities, Risks, Principles, and Recommendations. Minds Mach 28:689-707. https://doi.org/10.1007/s11023-018-9482-5

Frankfurt H (1971) Freedom of Will and Concept of a Person. J Philos 68:5-20

Friedman B, Nissenbaum H (1996) Bias in computer systems. ACM Trans Inf Syst 14:330-347. https://doi.org/10.1145/230538.230561

Gartner M (2021) Fit for the Future: A Pragmatic Account of Human Autonomy to Understand Emerging Issues in The Internet of Everything. In: forthcoming

Glasgow D (2021) Redefining what a map can be with new information and AI

Grifoni P, D’Ulizia A, Ferri F (2018) Context-Awareness in Location Based Services in the Big Data Era. pp 85-127

Hoffrage U, Marewski JN (2020) Social Scoring als Mensch-System-Interaktion. In: Social Credit
Rating. Springer Fachmedien Wiesbaden, Wiesbaden, pp 305-329

Hosanagar K, Krishnan R, Ma L (2008) Recomended for You: The Impact of Profit Incentives on the Relevance of Online Recommendations. In: ICIS 2008 Proceedings. p 31

Huang H, Gartner G, Krisp JM, et al (2018) Location based services: ongoing evolution and research agenda. J Locat Based Serv 12:63-93. https://doi.org/10.1080/17489725.2018.1508763

International Telecommunication Union (2020) Measuring digital development - Facts and figures

Koene A, Perez E, Carter CJ, et al (2015) Ethics of Personalized Information Filtering. pp 123-132

Kosters M, Van der Heijden J (2015) From mechanism to virtue: Evaluating Nudge theory. Evaluation 21:276-291. https://doi.org/10.1177/1356389015590218

Kupfer J (1987) Privacy, Autonomy, and Self-Concept. Am Philos Q 24:81-89

Lee MK (2018) Understanding perception of algorithmic decisions: Fairness, trust, and emotion in response to algorithmic management. Big Data Soc 5:205395171875668. https://doi.org/10.1177/2053951718756684

Martin K (2019) Ethical Implications and Accountability of Algorithms. J Bus Ethics 160:835-850. https://doi.org/10.1007/s10551-018-3921-3

Milano S, Taddeo M, Floridi L (2020) Recommender systems and their ethical challenges. AI Soc 35:957-967. https://doi.org/10.1007/s00146-02000950-y

Mittelstadt BD, Allo P, Taddeo M, et al (2016) The ethics of algorithms: Mapping the debate. Big Data Soc 3:205395171667967. https://doi.org/10.1177/2053951716679679

Owens J, Cribb A (2019) 'My Fitbit Thinks I Can Do Better!' Do Health Promoting Wearable Technologies Support Personal Autonomy? Philos Technol 32:23-38. https://doi.org/10.1007/s13347017-0266-2

Pew Research Center (2019) Smartphone Ownership Is Growing Rapidly Around the World, but Not Always Equally

Raper J, Gartner G, Karimi H, Rizos C (2007a) A critical evaluation of location based services and their potential. J Locat Based Serv 1:5-45. https://doi.org/10.1080/17489720701584069

Raper J, Gartner G, Karimi H, Rizos C (2007b) Applications of location-based services: a selected review. J Locat Based Serv 1:89-111. https://doi.org/10.1080/17489720701862184

Rappaport TS (1991) The wireless revolution. IEEE Commun Mag 29:52-71. https://doi.org/10.1109/35.109666 
Schiller J, Voisard A (2004) Location-Based Services. Elsevier

Schmidt A, Beigl M, Gellersen H-W (1999) There is more to context than location. Comput Graph 23:893-901. https://doi.org/10.1016/S00978493(99)00120-X

Shin D, Park YJ (2019) Role of fairness, accountability, and transparency in algorithmic affordance. Comput Human Behav 98:277-284. https://doi.org/10.1016/j.chb.2019.04.019

Sloan RH, Warner R (2018) When Is an Algorithm Transparent? Predictive Analytics, Privacy, and Public Policy. IEEE Secur Priv 16:18-25. https://doi.org/10.1109/MSP.2018.2701166

Sneddon A (2013) Autonomy, 1st edn. Bloomsbury Academic, London/New York

Special Rapporteur on the promotion and protection of the right to freedom of opinion and expression (2018) Seventy-third session Item 74 (b) of the provisional agenda** Promotion and protection of human rights: human rights questions, including alternative approaches for improving the effective enjoyment of human rights and fundamental freedoms

Stoljar N (2000) Autonomy and the feminist intuition. In: MacKenzie C, Stoljar. N (eds) Relational Autonomy: Feminist Perspectives on Autonomy, Agency and the Social Self. Oxford University Press, New York, pp 94-111

Taddeo M, Floridi L (2018) How AI can be a force for good. Science (80- ) 361:751-752. https://doi.org/10.1126/science.aat5991

Taib M (1990) Loophole allows bias in displays on computer reservations systems. Aviat Week Space Technol 132:137

Tene O, Polonetsky J (2013) Big Data for All: Privacy and User Control in the Age of Analytics. Northwest J Technol Intellect Prop 11:239-272

Thaler R, Sunstein C, Balz J (2013) Choice Architecture. In: The Behavioral Foundations of Public Policy. Princeton University Press, pp 428-439

Tsamados A, Aggarwal N, Cowls J, et al (2021) The ethics of algorithms: key problems and solutions. AI Soc. https://doi.org/10.1007/s00146-021-011548

Wall E, Stasko J, Endert A (2019) Toward a Design Space for Mitigating Cognitive Bias in Vis. In: 2019 IEEE Visualization Conference (VIS). IEEE, pp 111-115

Weiser M (1999) The computer for the 21 st century. ACM SIGMOBILE Mob Comput Commun Rev 3:3-11. https://doi.org/10.1145/329124.329126

Wicker SB (2012) The loss of location privacy in the cellular age. Commun ACM 55:60-68. https://doi.org/10.1145/2240236.2240255
Xu H, Gupta S (2009) The effects of privacy concerns and personal innovativeness on potential and experienced customers' adoption of location-based services. Electron Mark 19:137-149. https://doi.org/10.1007/s12525-009-0012-4

Yurur O, Liu CH, Sheng Z, et al (2016) ContextAwareness for Mobile Sensing: A Survey and Future Directions. IEEE Commun Surv Tutorials 18:68-93. https://doi.org/10.1109/COMST.2014.2381246

Zuboff S (2019) The Age of Surveillance Capitalism

Zuboff S (2015) Big other: Surveillance Capitalism and the Prospects of an Information Civilization. J Inf Technol 30:75-89. https://doi.org/10.1057/jit.2015.5 